\title{
CryoTEM with a Cold Field Emission Gun That Moves Structural Biology into a New Stage
}

Takayuki Kato $^{1}$, Fumiaki Makino ${ }^{1}$, Takanori Nakane ${ }^{2}$, Naoya Terahara ${ }^{1}$, Takeshi Kaneko ${ }^{3}$, Yuko

$\mathrm{Shimizu}^{3}$, Sohei Motoki ${ }^{3}$, Isamu Ishikawa ${ }^{3}, K_{\text {Koji Yonekura }}^{4}$ and Keiichi Namba ${ }^{1,4,5^{*}}$

1. Graduate School of Frontier Biosciences, Osaka University, Suita, Osaka, Japan.

2. MRC Laboratory of Molecular Biology, Cambridge, UK.

3. JEOL, Akishima, Tokyo, Japan

4. RIKEN SPring-8 Center, Sayo-cho, Hyogo, Japan.

5. RIKEN Center for Biosystems Dynamics Research, Suita, Osaka, Japan.

* Corresponding author: keiichi@fbs.osaka-u.ac.jp

Structural Biology, which reveals three-dimensional (3D) structures and the dynamics of biological macromolecules and complexes, is fundamental not only for life sciences but also for medical sciences and drug design, because all the biological functions, such as signal and energy transductions, and their mechanisms are determined by finely designed 3D arrangements of constituent atoms, their motions and interactions with specific partner molecules. X-ray crystallography and NMR are powerful tools but electron cryomicroscopy (cryoEM) is now attracting much attention as a tool for high-resolution structural analysis partly due to recent development of CMOS-based, highly-sensitive, fast image detectors that allows single electron counting and movie mode imaging [1]. Together with development of powerful image analysis software packages, such as RELION [2,3], cryoEM image analysis can now achieve near atomic resolution in 3D structural analysis of biological macromolecules with a very small amount of solution samples, as small as a few ten of $\mu$ grams, without the need of crystallization. All one needs is to apply a few $\mu$ l sample solution on to a holey carbon grid, blot most of it to make thin solution films within each carbon hole and flash freeze the entire grid to embed biomolecules in vitreous ice thin film in a frozen hydrated native state. Transmission electron microscopes with a stable cryo specimen stage cooled conventionally by liquid $\mathrm{N}_{2}$ (cryoTEM) are used to record images of biomolecules embedded in many different orientation in vitreous ice film. One must keep the electron dose as low as possible to avoid radiation damage, but this leads to a poor image quality due to a low signal to noise ratio $(\mathrm{S} / \mathrm{N})$. To recover high-resolution structural information in $3 \mathrm{D}$ image reconstruction, one needs to collect thousands of images to increase the $\mathrm{S} / \mathrm{N}$ by aligning and averaging a large number of particle images. That is why it is essential to use a highly sensitive, fast detector with the capability of motion correction for movie mode images to reduce blurs that would occur in integrating image recording. It is also important to use a highly automated, stable cryoTEM with high-resolution electron optics for high throughput data collection. Titan Krios (Thermo Fisher Scientific) has become the most popular cryoTEM because it fulfills such requirements for users to achieve near atomic resolution nearly routinely.

JEOL produced a TEM/STEM called ARM (Atomic Resolution Microscope) with an extremely highresolution electron optics designed for material science studies, and we have developed a cryoTEM called CRYO ARM by adapting the electron optics of ARM. Also developed are an auto specimen grid loader, a cryo workstation for frozen grid transfer to the autoloader, a liquid $\mathrm{N}_{2}$-cooled side-entry specimen stage, a user friendly TEM control software and an automated data collection software, JADAS. The autoloader can accommodate up to 12 grids and allow grid replacement from 1 to 4 at a time. The TEM control software is featured with auto alignment of optics for parallel beam and coma 
free imaging, and together with JADAS, any users can collect high resolution images in high throughput. The last and most advanced feature for high resolution imaging is a stable cold field emission electron gun (FEG). The coherence of the electron beam is markedly higher than the one from a Schottky type thermal FEG because the energy spread of the former is nearly half of the latter.

In mid 2017 we used a prototype CRYO ARM 200 with a thermal FEG operated at $200 \mathrm{kV}$ and a Gatan K2 camera and produced a $2.41 \AA$ resolution density map of $\beta$-galactosidase from 98,158 particles extracted from about 2,500 images collected over 3 days [4]. Now by using CRYO ARM 300, the first commercial version installed at RIKEN SPring-8 Center, we have achieved $1.54 \AA$ resolution in the 3D reconstruction of mouse apoferritin structure from 120,295 particle images extracted only from 840 images collected over one day. The map shows features of truly atomic resolution (Figure 1). It is worthy of note that merely the first 56 images of the full data set processed as a preliminary test produced a $1.76 \AA$ resolution map. The B-factor of the cryoEM images is 46.9 , which is the smallest one obtained to date. These results suggest that the cold FEG is quite effective in enhancing high resolution image signal, and a stable cold FEG, such as the one used in CRYO ARM, will be a powerful tool for atomic resolution structural analysis by single particle image analysis.

\section{References:}

[1] X Li et al., Nature Methods 10 (2013), p. 584.

[2] SHW Scheres, Journal of Structural Biology 180 (2012), p. 519.

[3] J Zivanov et al., Elife 7 (2018), e42166.

[4] T Kato et al., EMDB ID: 6840.

[5] The authors acknowledge all the JEOL engineers and staffs for their efforts in the development of CRYO ARM, Haruaki Yanagisawa and Masahide Kikkawa for the plasmid of mouse apoferritin, and funding from AMED BINDS to TK and KN, AMED CiCLE to JEOL, and JSPS KAKENHI Grant number 25000013 to KN. Takayuki Kato, Fumiaki Makino and Takanori Nakane contributed equally.
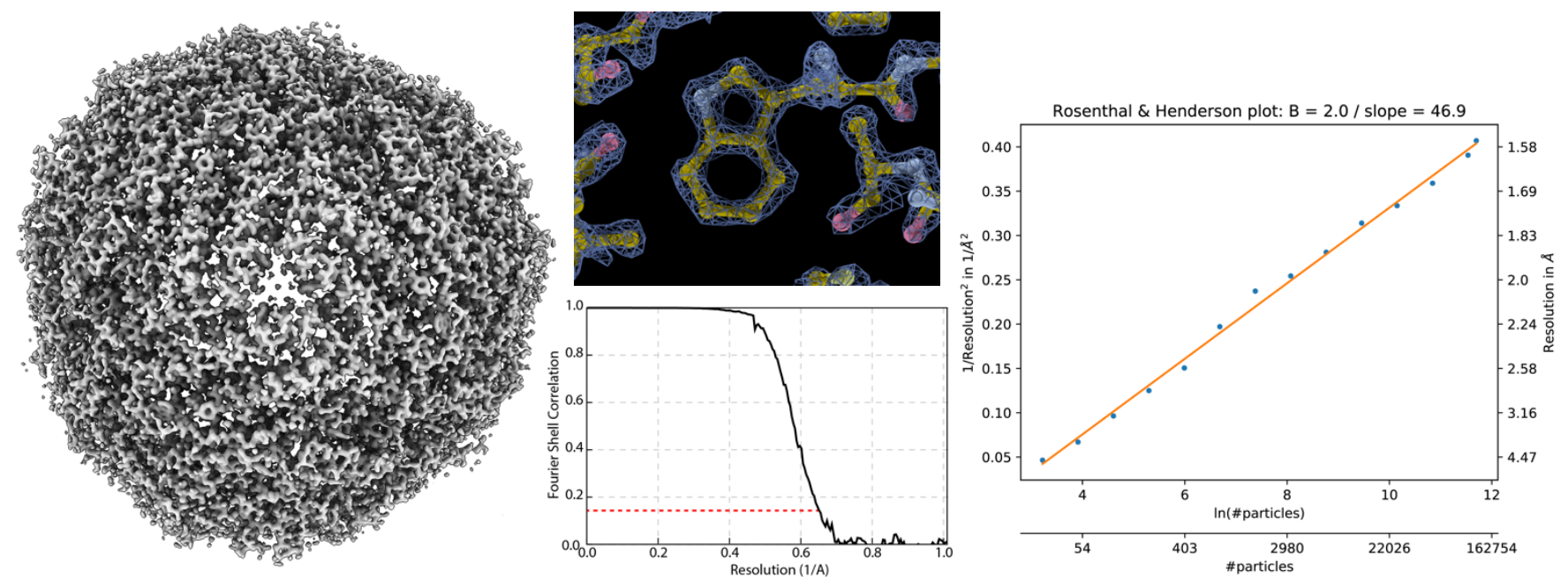

Figure 1. $3 \mathrm{D}$ density maps of mouse apoferritin at $1.54 \AA$ resolution. Left: the entire structure of apoferritin with the 3 -fold axis in front. Middle: a density map of tryptophan showing clear holes in its five- and six-membered rings (upper panel) and the Fourier shell correlation curve (lower panel). Right: Rosenthal \& Henderson plot showing the B-factor of 46.9, the smallest one estimated for cryoEM images to date. 